\title{
Study on Micron Porous Copper Prepared by Physical Vacuum Dealloying
}

\author{
Yi-Bin $\operatorname{Ren}^{1} \cdot$ Yu-Xia Sun ${ }^{2} \cdot$ Ke Yang ${ }^{1}$
}

Received: 30 June 2016/Revised: 8 September 2016/Published online: 31 October 2016

(C) The Chinese Society for Metals and Springer-Verlag Berlin Heidelberg 2016

\begin{abstract}
Porous copper was prepared successfully by physical vacuum dealloying method using the CuZn alloy precursors (Cu30Zn70, Cu40Zn60 and Cu50Zn50 alloys). The micron porous copper showed a three-dimensional continuous porous structure with 1-5 $\mu \mathrm{m}$ pore size. With the increase of the $\mathrm{Zn}$ content in the $\mathrm{CuZn}$ alloy, the pore structure of the porous copper was more uniform and ordered. Temperature was the key factor for physical dealloying, and the optimized temperature was $500{ }^{\circ} \mathrm{C}$ for the CuZn alloy. The pores would fuse and disappear when the temperature was over $500{ }^{\circ} \mathrm{C}$. Physical vacuum dealloying was an effective preparation method for porous copper, which can be used to prepare other porous metals based on the sublimation and the Kirkendall effect.
\end{abstract}

KEY WORDS: Dealloying; Sublimation; Kirkendall effect; Porous copper; $\mathrm{Cu}-\mathrm{Zn}$ alloy

\section{Introduction}

Recently, porous metals have attracted much attention for applications such as lightweight structure, energy absorber, heat exchanger, biomaterial and catalysis. So far, various fabrication methods for porous metals have been developed [1-3], including foaming by gas bubbling, metal deposition on polyurethane foam by electroplating, powder sintering, dealloying.

Porous copper is an interesting electrode material for the electrolysis process, and the porous copper electrode was prepared by selective dissolution of aluminum from $\mathrm{CuAl}_{2}$ alloy in $\mathrm{NaOH}$ solution [4]. Nakajima [1] investigated the fabrication of porous copper by unidirectional

Available online at http://link.springer.com/journal/40195

Yi-Bin Ren

ybren@imr.ac.cn

1 Institute of Metal Research, Chinese Academy of Sciences, Shenyang 110016, China

2 Shenyang Aerospace University, Shenyang 110136, China solidification under hydrogen, and the pore diameter ranged from $5 \mu \mathrm{m}$ up to $5 \mathrm{~mm}$. The lost carbonate sintering (LSC) process was also used for manufacturing open cell copper foams [5], with a size ranged from $53 \mu \mathrm{m}$ up to $1.5 \mathrm{~mm}$.

Additionally, porous copper can be prepared by electrochemical dealloying of the $\mathrm{CuZn}$ alloy in many kinds of solutions [6, 7]. Porous copper prepared by dealloying was usually nanoporous copper (NPC), and several alloy systems including $\mathrm{AlCu}, \mathrm{CuZn}, \mathrm{CuZr}, \mathrm{MnCu}$ and $\mathrm{MgCu}$ have been successfully developed [6-11].

Dealloying has demonstrated to be very efficient in producing three-dimensional (3D) porous metals $[12,13]$. At present, the NPCs were prepared usually by selective dissolution of one metal element which was out of a binary alloy based on chemical or electrochemical principle. Recently, we have developed a novel physical dealloying method to prepare micron porous metals in vacuum environment [14-16].

Physical vacuum dealloying (PVD), or physical sublimation dealloying (PSD), is a common process by subliming one or more metal components from an alloy to form a porous metal. The precursor alloy must contain 
more than one metal element with a high vapor pressure, and at a specified temperature in vacuum environment, the matrix metal will undergo sublimation and diffusion to form voids and finally form a 3D micron porous structure with interconnected ligaments. In this study, a micron porous copper was fabricated using $\mathrm{Cu} 30 \mathrm{Zn70}, \mathrm{Cu} 40 \mathrm{Zn} 60$ and Cu50Zn50 alloys by physical dealloying method and the microtopography and some influence factors were studied.

\section{Experimental}

The Cu30Zn70, Cu40Zn60 and Cu50Zn50 precursor alloys were fabricated by heating $\mathrm{Cu}$ powders and $\mathrm{Zn}$ blocks with atomic ratio of 3:7, 4:6 and 5:5 in a nitrogen atmosphere, respectively. The temperature of furnace containing $\mathrm{Cu}$ and $\mathrm{Zn}$ was raised to $900 \pm 50{ }^{\circ} \mathrm{C}$ and held for $0.5 \mathrm{~h}$; then the melt was cast in the graphite crucible and finally cooled down to ambient temperature. The samples with a dimension of $5 \mathrm{~mm} \times 5 \mathrm{~mm} \times 0.5 \mathrm{~mm}$ were sectioned from ingots by wire-electrode cutting, and the samples were polished with $\mathrm{SiC}$ papers of 1000 grit size and rinsed with absolute ethyl alcohol before physical dealloying.

The physical sublimation dealloying experiments were performed, respectively, at 500, 600 and $700{ }^{\circ} \mathrm{C}$ in dynamic high vacuum environment for $3 \mathrm{~h}$, and the vacuum during experiment was kept under $10 \mathrm{~Pa}$ or lower. Three different samples were used for each test condition, and the measured results of the pore size distribution were average. The surface morphology, composition, and crystal structure of the physical dealloyed samples were characterized by scanning electron microscopy (SEM) equipped with energy dispersive X-ray spectroscopy (EDS) and $\mathrm{X}$-ray diffraction (XRD) respectively.

\section{Results}

After the physical vacuum dealloying processes, colors of all the samples were changed from silver-white to red under naked eye observation. Figure 1 shows the XRD patterns of the as-prepared Cu50Zn50, Cu40Zn60 and $\mathrm{Cu} 30 \mathrm{Zn} 70$ precursor alloys and the micron porous copper from Cu30Zn70 alloy at $500{ }^{\circ} \mathrm{C}$. Prior to dealloying, the samples of Cu50Zn50, Cu40Zn60 and Cu30Zn70 alloys contained different single phases with diffraction peaks, respectively, corresponding to $\mathrm{Cu} 1.05 \mathrm{Zn} 0.95$ (JCPDS Card No. 65-9060), CuZn (JCPDS Card No. 65-6321) and Cu5Zn8 (JCPDS Card No. 65-3157), as shown in Fig. 1ac, which reveals that the Cu50Zn50, Cu40Zn60 and Cu30Zn70 precursor alloys should mainly consist of Cu1.05Zn0.95, CuZn and Cu5Zn8 phases, respectively.

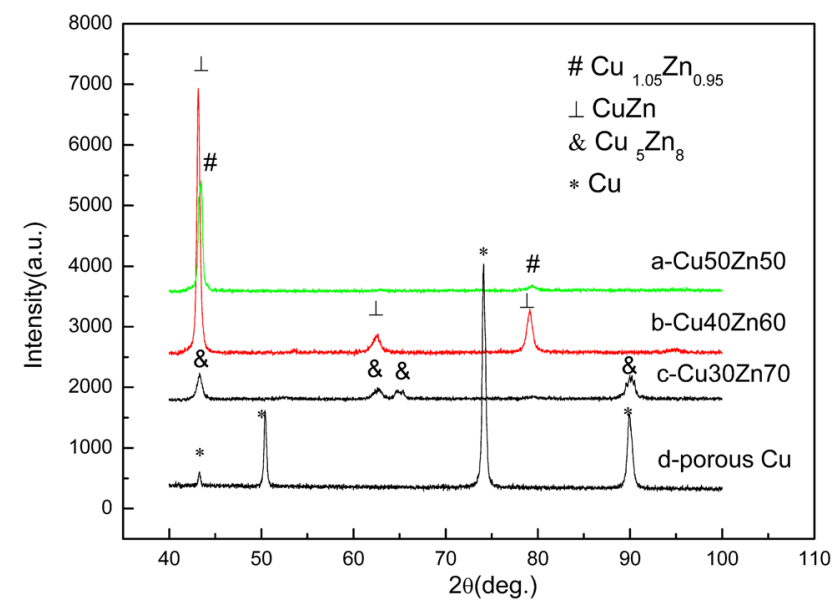

Fig. 1 XRD patterns of Cu50Zn50, Cu40Zn60 and Cu30Zn70 precursor alloys before $(a-c)$, after $(d)$ physical vacuum dealloying

After the physical vacuum dealloying, the polycrystalline pure $\mathrm{Cu}$ phase (JCPDS Card No. 65-9743) can be seen, as shown in Fig. 1d.

Figures 2, 3 and 4 show the surface SEM images of the Cu50Zn50, Cu40Zn60 and Cu30Zn70 alloys after physical vacuum dealloying at $500{ }^{\circ} \mathrm{C}$, respectively. Figures $2 \mathrm{a}$, 3a and $4 \mathrm{a}$ are the as-prepared micron porous copper, and Figs. $2 b, 3 b$ and $4 b$ are the partial enlarged view of Figs. 2a, 3a and 4a. EDS analyses (Figs. 2c, 3c, 4c) show that almost all $\mathrm{Zn}$ on the surface of alloys were complete sublimated. The widths of channel and ligament of micron porous copper of Cu50Zn50 alloy are $3 \pm 1 \mu \mathrm{m}$, and the diameter of pore is $1 \pm 1 \mu \mathrm{m}$.

With increasing the $\mathrm{Zn}$ content in the alloy, the widths of the channel and ligament of the micron porous copper were reduced from $\mathrm{Cu} 40 \mathrm{Zn} 60$ alloy of $1 \pm 0.5 \mu \mathrm{m}$ to $\mathrm{Cu} 30 \mathrm{Zn} 70$ alloy of $0.5 \pm 0.5 \mu \mathrm{m}$, and the pore size increased from $2 \pm 1$ to $5 \pm 1 \mu \mathrm{m}$. In addition, the micron porous copper from $\mathrm{Cu} 30 \mathrm{Zn} 70$ alloy exhibited a well-developed three-dimensional continuous porous structure, and its relative density was about $30 \%$ of bulk Cu density, which was determined by the measured mass and calculated volume, demonstrating the complete sublimation of $\mathrm{Zn}$ and the existence of holes in the porous copper.

\section{Discussion}

The method used in this study is known as physical dealloying because the process just includes two physical reactions, sublimation and diffusion, which played a dominant role during the whole process. In brief, the formation of porous structure is a result of a competition between the selective sublimation and diffusion process of the alloy components. In addition to the CuZn alloy, this 

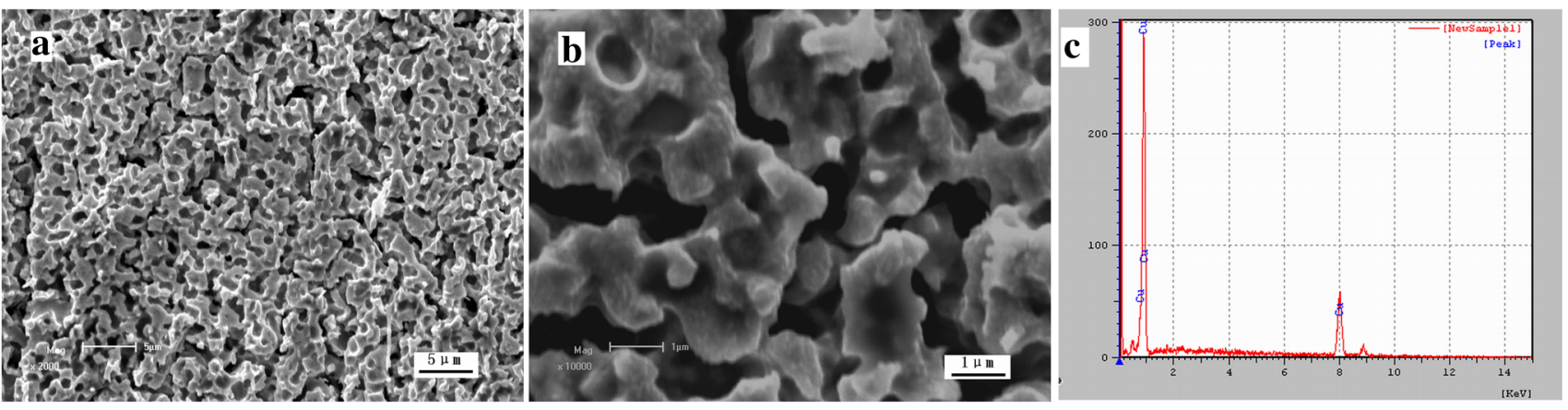

Fig. 2 SEM images and EDS analysis of Cu50Zn50 alloy after physical vacuum dealloying
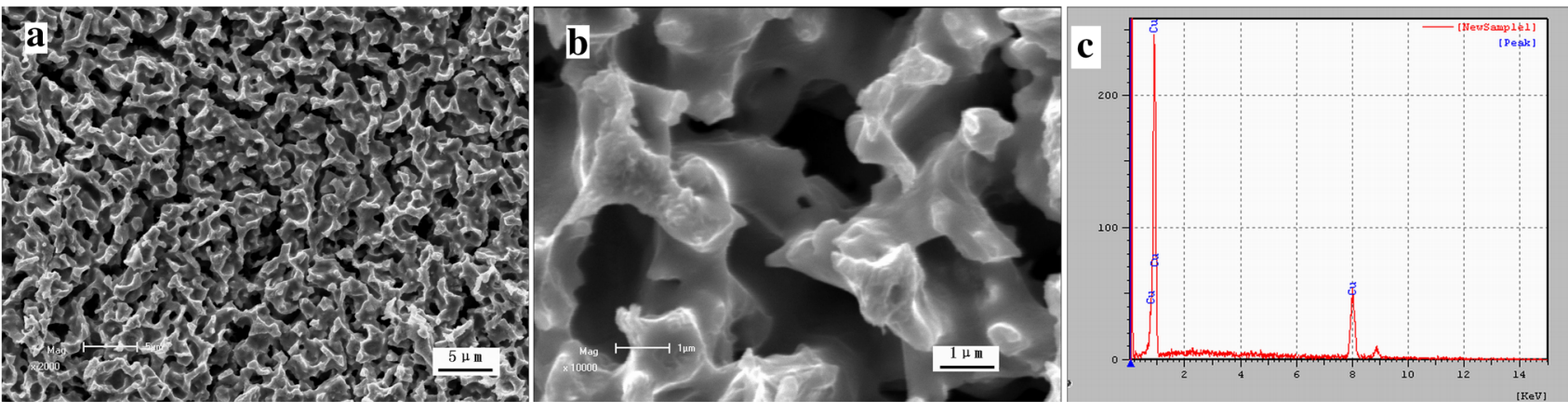

Fig. 3 SEM images and EDS analysis of Cu40Zn60 alloy after physical vacuum dealloying
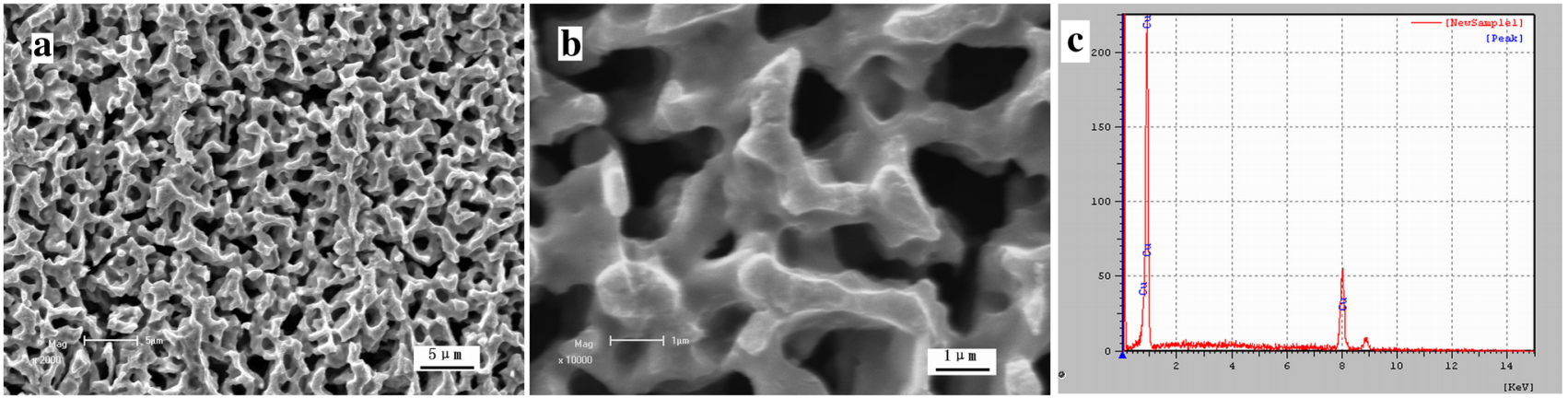

Fig. 4 SEM images and EDS analysis of Cu30Zn70 alloy after physical vacuum dealloying

method can be also applied for the preparation of other porous metals [14]. In the present study, this method has demonstrated its effectiveness by three CuZn alloys, and the $\mathrm{Zn}$ was the key element in CuZn alloy to form the micron porous copper. Firstly, $\mathrm{Zn}$ has a high saturated vapor pressure, for instance 1 Torr at $487{ }^{\circ} \mathrm{C}$ [17], which makes it full sublimation at high temperature in vacuum environment. Secondly, the diffusion rate of $\mathrm{Zn}$ is much greater than that of $\mathrm{Cu}$ in the $\mathrm{CuZn}$ alloy [18]. Therefore, $\mathrm{Zn}$ can be rapidly sublimated from the surface of $\mathrm{CuZn}$ alloy at $500{ }^{\circ} \mathrm{C}$ in vacuum environment. Thirdly, according to Kirkendall effect, a large amount of vacancies or voids can be formed in the $\mathrm{CuZn}$ alloy because of the differential diffusion between $\mathrm{Cu}$ and $\mathrm{Zn}$. Therefore the pores will be formed and start from the surface of the CuZn alloys because of their great difference in the saturated vapor pressures, then the sublimation and the differential diffusion affected mutually to trigger dealloying to form the porous copper.

The Kirkendall effect is a classical phenomenon in metallurgy, which is a consequence of different diffusivities of atoms in a diffusion couple, causing a supersaturation of lattice vacancies. This supersaturation may lead to a condensation of extra vacancies in the form of so-called Kirkendall voids, and these Kirkendall voids are generally considered as a nuisance because they deteriorate the properties of the alloy. In contrast, the Kirkendall effect has been positively used as a new fabrication route to produce porous metal. The nanotubes and hollow nanoparticles based on the Kirkendall effect have been prepared, and 

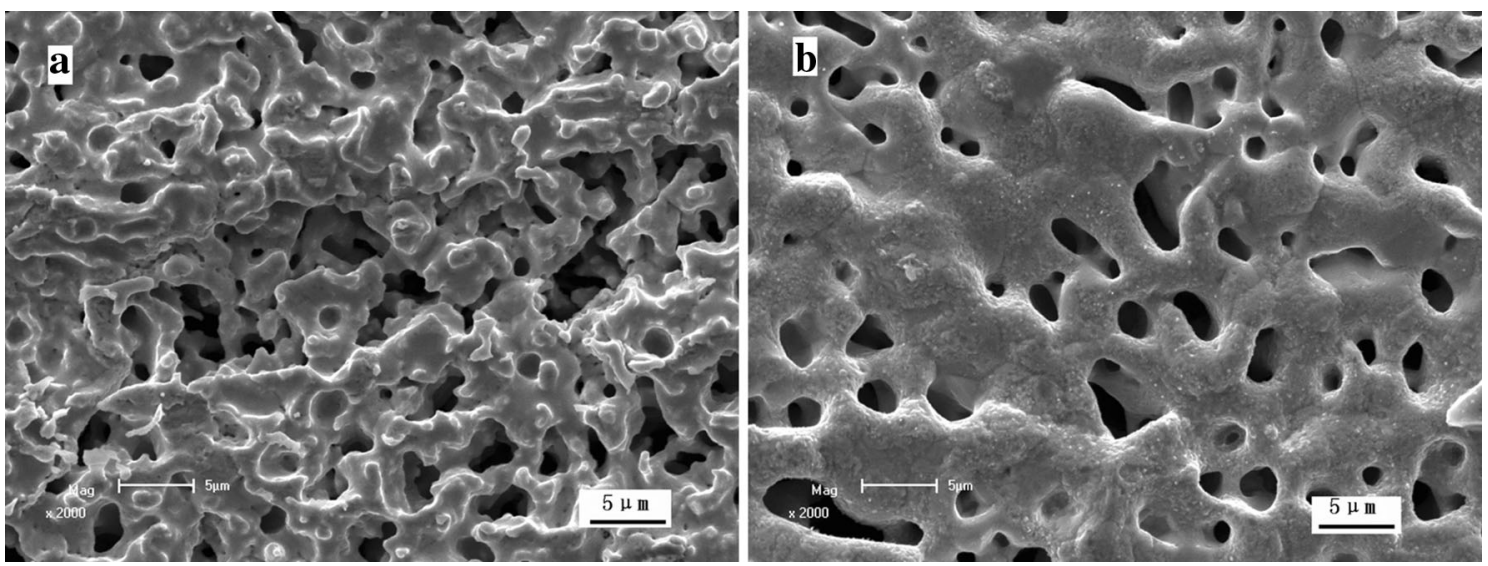

Fig. 5 SEM images of Cu30Zn70 alloy after physical dealloying at $600(\mathbf{a})$, at $700{ }^{\circ} \mathrm{C}(\mathbf{b})$

such an effect has become an interesting synthesis route to produce nanoscale hollow structures of various material systems $[19,20]$. In this study, the combined effect of the sublimation and the differential diffusion (Kirkendall effect) of $\mathrm{Zn}$ in the CuZn alloy is used to produce the porous copper.

In our previous study, the temperature was also the key factor for physical dealloying [15], which was not only involved in the sublimation rate, but also affected the diffusivity of $\mathrm{Zn}$ in the CuZn alloy. Figure 5 shows the SEM images of the $\mathrm{Cu} 30 \mathrm{Zn} 70$ alloy after physical vacuum dealloying at 600 and $700{ }^{\circ} \mathrm{C}$, respectively. With increasing the vacuum heat treatment temperature, the pores of porous copper were fused gradually and even this alloy had relative large pores at $500{ }^{\circ} \mathrm{C}$. According to the Kirkendall effect, the diffusivity of $\mathrm{Zn}$ would be increased with the increase in the temperature (the intrinsic diffusivity of $\mathrm{Zn}$ is about 2.5 times than that of $\mathrm{Cu}$ at $785{ }^{\circ} \mathrm{C}$ [20]), and the Kirkendall voids should be enlarged with increasing the temperature, but in fact they partly merged together. As we know, the diffusivity of $\mathrm{Cu}$ was also increased with increasing the temperature, and the most lattice vacancies formed in the migration of $\mathrm{Zn}$ would be occupied by $\mathrm{Cu}$ atoms, which should be more active at higher temperature. Therefore, many small Kirkendall voids disappeared. So the physical dealloying can occur at an optimized temperature in vacuum environment for the some special alloys, and the temperature was about $500{ }^{\circ} \mathrm{C}$ for the $\mathrm{CuZn}$ alloy in the present study.

\section{Conclusion}

This study showed that the micron porous copper could be prepared by physical vacuum dealloying of the CuZn alloy. The micron porous copper showed a three-dimensional continuous porous structure with $1-5 \mu \mathrm{m}$ pore size. With the increase of the $\mathrm{Zn}$ content in the CuZn alloy, the pore structure of the porous copper becomes more even and orderly, and the temperature was the key factor for the physical dealloying process. The porous structure would be fused and disappeared when the treatment temperature was higher than the optimized temperature of $500{ }^{\circ} \mathrm{C}$.

Acknowledgments This work was supported by the National Natural Science Foundation of China (Grant No. 31370976) and the National Basic Research Program of China (Grant No. 2012CB619101).

\section{References}

[1]. H. Nakajima, Prog. Mater. Sci. 52, 1091 (2007)

[2]. P. Liu, B. Yu, A. Hu, K. Liang, S. Gu, J. Mater. Sci. Technol. 18, 299 (2002)

[3]. B. Li, X. Lu, Acta Metall. Sin. (Engl. Lett.) 27, 338 (2014)

[4]. A. Smith, D. Trimm, Annu. Rev. Mater. Res. 35, 127 (2005)

[5]. Y. Zhao, T. Fung, L. Zhang, Scr. Mater. 52, 295 (2005)

[6]. R. Mao, S. Liang, X. Wang, Q. Yang, B. Han, Corros. Sci. 60, 231 (2012)

[7]. Y. Lin, C. Tai, I. Sun, J. Electrochem. Soc. 154, D316 (2007)

[8]. J. Li, H. Jiang, N. Yu, C. Xu, H. Geng, Corros. Sci. 90, 216 (2015)

[9]. C. Zhao, Z. Qi, X. Wang, Z. Zhang, Corros. Sci. 51, 2120 (2009)

[10]. J. Hayes, A. Hodge, J. Biener, A. Hamza, K. Sieradzki, J. Mater. Res. 21, 2611 (2006)

[11]. H. Lu, Y. Li, F. Wang, Scr. Mater. 56, 165 (2007)

[12]. J. Erlebacher, M. Aziz, A. Karma, N. Dimitrov, K. Sieradzki, Nature 410, 450 (2001)

[13]. A. Chen, S. Shi, Y. Qiu, X. Xie, H. Ruan, J. Gu, D. Pan, Microporous Mesoporous Mater. 202, 50 (2015)

[14]. Y. Ren, Y. Sun, K. Xiao, K. Yang, Chinese invention patent. 201410181273.0 (2014)

[15]. Y. Sun, Y. Ren, K. Yang, Mater. Lett. 165, 1 (2016)

[16]. Y. Sun, Y. Ren, Vacuum 122, 215 (2015)

[17]. R.E. Honig, D.A. Kramer, RCA Rev. 30, 285 (1969)

[18]. E. Kirkendall, L. Thomassen, C. Upthegrove, Trans. AIME 133, 186 (1939)

[19]. Y. Yin, R. Rioux, C. Erdonmez, S. Hughes, G. Somorjai, A. Alivisatos, Science 304, 711 (2004)

[20]. H. Fan, U. Gösele, M. Zacharias, Small 3, 1660 (2007) 